\title{
Environmental assessment of two different crop systems in terms of biomethane potential production
}

\author{
Jacopo Bacenetti, Alessandra Fusi *, Marco Negri, Riccardo Guidetti, Marco Fiala \\ Department of Agricultural and Environmental Sciences, Production, Landscape, Agroenergy, Università degli Studi di Milano, Via G. Celoria 2, 20133 Milan, Italy
}

H I G H L I G H T S

- Environmental impact of two crop systems was evaluated

- Biomethane specific production tests were carried out

- Alternative scenarios (different yields and crop management) were assessed

- Maize single crop obtains the better environmental performance

- Critical factors are: fertilizer and diesel fuel emissions and diesel fuel production

\section{A R T I C L E I N F O}

\section{Article history:}

Received 22 April 2013

Received in revised form 23 July 2013

Accepted 29 July 2013

Available online $\mathrm{xxx}$

\section{Editor: Charlotte Poschenrieder}

\section{Keywords:}

Energy crops

Anaerobic digestion

Life cycle assessment

Environmental sustainability

Biogas

\begin{abstract}
A B S T R A C T
The interest in renewable energy sources has gained great importance in Europe due to the need to reduce fossil energy consumption and greenhouse gas emissions, as required by the Renewable Energy Directive (RED) of the European Parliament. The production of energy from energy crops appears to be consistent with RED. The environmental impact related to this kind of energy primarily originates from crop cultivation. This research aimed to evaluate the environmental impact of different crop systems for biomass production: single and double crop. The environmental performances of maize and maize plus wheat were assessed from a life cycle perspective. Two alternative scenarios considering different yields, crop management, and climatic conditions, were also addressed. One normal cubic metre of potential methane was chosen as a functional unit. Methane potential production data were obtained through lab experimental tests. For both of the crop systems, the factors that have the greatest influence on the overall environmental burden are: fertilizer emissions, diesel fuel emissions, diesel fuel production, and pesticide production. Notwithstanding the greater level of methane potential production, the double crop system appears to have the worse environmental performance with respect to its single crop counterpart. This result is due to the bigger quantity of inputs needed for the double crop system. Therefore, the greater amount of biomass (silage) obtained through the double crop system is less than proportional to the environmental burden that results from the bigger quantity of inputs requested for double crop.
\end{abstract}

(c) 2013 Elsevier B.V. All rights reserved.

\section{Introduction}

The use of renewable sources for energy production is considered to be a potential solution for reducing the environmental problems derived from fossil fuels (Cherubini et al., 2009; Appels et al., 2011; González-García et al., 2012a). However, the environmental impacts of agricultural production systems have raised concern from national as well as international points of view. According to the Intergovernmental Panel on Climate Change, agriculture contributes a share of $13.5 \%$ to global anthropogenic greenhouse gas (GHG) emissions (Bachmaier et al., 2010; Carozzi et al., 2013).

\footnotetext{
* Corresponding author. Tel.: + 3902 50316873; fax: + 390250316845 .

E-mail address: alessandra.fusi@unimi.it (A. Fusi).
}

In Europe, the interest in Renewable Energy Sources (RES) has strongly increased due to the need to reduce fossil energy consumption and greenhouse gas (GHG) emissions, as indicated by DIRECTIVE 2009/ 28/EC (RED) (European Parliament Council, 2010, 2009). According to RED, Italy should be able to produce $17 \%$ of primary energy using RES by 2020 (in 2009, energy production from RES was already at 8.86\%, and in 2010, it was $>10 \%$ ). In particular, RES should produce 100 TWh/year, covering 26\% of electric consumption (Ministero dello Sviluppo Economico, 2012).

Energy crops and corresponding derived bioenergy production are expected to bring environmental, social, and economic benefits. Several studies have reported benefits in terms of the reduction of greenhouse gas emissions, air pollution, acidification, or eutrophication (Brentrup et al., 2004; Buratti and Fantozzi, 2010; Kimming et al., 2011; Bacenetti et al., 2012a; González-García et al., 2012b). However, the 
environmental impacts strongly depend on the crop cultivation (Fazio and Monti, 2011; González-García et al., 2012c; Uchida and Hayashi, 2012).

For the achievement of European Union (EU) objectives, the anaerobic digestion ( $A D$ ) of energy crops and agro-industrial by-products and/ or wastes appears to be one of the most promising agro-energy chains (Angelidaki and Ellegaard, 2003; Edelmann et al., 2005; Clemens et al., 2006; Wulfa et al., 2006; Börjesson and Berglund, 2007; Jury et al., 2010; Patterson et al., 2011; Capponi et al., 2012). Among all possible solutions, $A D$ represents one of the most promising ways to use RES (Börjesson and Mattiasson, 2008).

Agricultural raw materials, such as straw and manure, are commonly used for biogas production (Del Prado et al., in press). Livestock activities are in fact widespread in Italy and there is, consequently, a great availability of manure. Nevertheless, the main feeding materials for digesters are often represented by cereal silages (of maize, wheat, and triticale, in particular) (Lansche and Müller, 2012).

In 2010, in Germany which is the country with the most significant biogas production (about 7000 biogas plants), more than one-third of the maize area $(2,282,000 \mathrm{ha})$ was used for bioenergy production (Dressler et al., 2012). In Italy, about 1000 agricultural biogas plants are currently (December 2012) in function (374 located in Lombardy), for a global electrical power of $756 \mathrm{MW}$ (Bacenetti et al., 2013); about $10 \%$ of the total maize area $(1,172,000 \mathrm{ha})$ was specifically cultivated for biogas purposes (Casati, 2013).

Although detailed information concerning the silage used for the AD plants is not available, in the areas in which biogas production is more widespread an increase in biomass prices and the value of land has taken place (Povellato, 2011).

The production of cereal ensilage can be carried out mainly with two different crop systems: the single crop system with sorghum or maize FAO classes 600 or 700 , or the double crop system with winter cereals (wheat, barley or triticale) followed by maize FAO classes 300, 400 or 500. In Lombardy, the maize hybrids FAO Class 700 are the most used for energy production in the single crop system, while maize hybrids FAO Classes 400 - 500 can be suitable after the harvesting of winter cereals, when the double culture system is chosen. However, the choice between the single crop or the double crop system must be carefully evaluated. In the double crop system, despite a moderated increase of production, field operations as well as production factors used (fertilizers, seeds, pesticide) are approximately double that in maize 700. Consequently, double crop systems involve higher economic costs.

In addition, it must be considered that the possibility for using double crop systems is linked to climatic conditions. The key factor is the speed with which tillage operations and sowing of maize 500 are performed after the harvest of the wheat. In years with a rainy spring this might not be allowed, or it could force the choice of maize hybrids with a shorter vegetative cycle (for example, maize class 300 or 400) instead of maize class 500 (which shows higher biogas production). Environmental effects caused by energy crop cultivation come not only from field operations but also from raw material (fuels, fertilizers, and pesticides) extraction, production and transportation (Scacchi et al., 2010). Therefore, in order to perform a complete evaluation of the system, all of these aspects must be taken into account.

Life cycle assessment (LCA) is a methodology that aims to analyze products, processes, or services from an environmental perspective [ISO 14040, 2006] (Guinée et al., 2002; ISO, 2006), providing a useful and valuable tool for agricultural system evaluation (Audsley, 1997; Brentrup et al., 2001; IPCC, 2006; Finnveden et al., 2009; Fiala and Bacenetti, 2011) as well as for energy crops (Gasol et al., 2009; Bacenetti et al., 2012b; González-García et al., 2012b).

The aim of this study was to analyze the environmental performances of two different crop systems (single and double crop) cultivated in Northern Italy, used for producing biomass for energy purposes. The LCA method was chosen to perform the environmental analysis.

\section{Materials}

\subsection{Goal and scope definition}

The environmental performances of a single crop (maize class 700 or maize 700) and a double crop (maize class 500 or maize 500 plus wheat) were compared in terms of methane potential production. Moreover, the most critical stages for both crop systems under study throughout the life cycle were identified.

The choice of the selected biomasses was due to their diffusion in the Lombardy region. Maize 700 is the best maize hybrid for single crop cultivation in the Po Valley area while Maize 500 is the most suitable maize hybrid for second sowing after a winter cereal. Therefore, in these climatic conditions, maize 700 (as a single crop) and the cultivation of wheat followed by maize 500 (as a double crop) are the two solutions which allow the better exploitation of the growing season. For this reason these two cropping systems were evaluated using the LCA methodology.

\subsection{Description of the cropping systems under assessment}

Wheat (Triticum spp. L.), which is a winter crop, and maize (Zea mais L.), which is a summer crop, were analyzed. Two FAO maize classes, in particular, were considered: maize 700 and maize 500 .

Two different crop systems were taken into account:

1. Single crop: maize 700 only;

2. Double crop: wheat followed by maize 500 .

For the double crop system, the seed bed preparation for maize cultivation is realized immediately after harvesting the wheat.

Cultivation of both analyzed crops is located in the Po Valley area, district of Milan, Lombardy region (Italy). The local climate is characterized by an average annual temperature of $12.7^{\circ} \mathrm{C}$, and rainfall is mainly concentrated in autumn and spring (average annual precipitation is equal to $745 \mathrm{~mm}$ ).

Field operations can be divided into four main steps: (1) soil tillage; (2) crop growth; (3) biomass harvesting and transport; and (4) biomass ensilage. Operations included in each step are shown in Fig. 1. Basically, the two crop systems differ in terms of the land occupation time: 5 and 12 months per year for single and double crop systems, respectively. There are differences between maize and wheat and also between maize 700 and maize 500, with regard to applied fertilizers and pesticides rates, seeds and water, and diesel fuel amounts. Field and ensilage operations for the three crops under study are described in the following subsections and reported as supplementary material.

\subsubsection{Maize}

Maize is the most widespread summer crop in Italy; in 2011, about 1 million hectares were cultivated [24\% in Lombardy] (ISTAT, 2011). In May, before ploughing, the soil will have been fertilized with digestate at rates of 45 and $85 \mathrm{tha}^{-1}$ for the maize 500 and 700 , respectively. After ploughing, always in May, the soil is harrowed, sown, and treated with herbicides [Lumax, $4 \mathrm{~kg} \mathrm{ha}^{-1}$ ]. The sowed seeds range from 70,000 $\left(19 \mathrm{~kg} \mathrm{ha}^{-1}\right)$ to $77,000\left(20 \mathrm{~kg} \mathrm{ha}^{-1}\right)$ seeds ha ${ }^{-1}$ depending on maize classes. In addition to the digestate spreading, mineral fertilization, using potassium- and phosphorous-based fertilizers, is carried out for maize 500 between the ploughing and harrowing. Chemical weed control is carried out twice in June using $1 \mathrm{~kg} \mathrm{ha}^{-1}$ of Dual. In the same month, hoeing and mineral fertilization with urea are performed.

For maize, irrigation can increase and make the yield steady. After the top fertilization, irrigation is performed between four and five times for maize 500 and 700, respectively, in July and August. The water volume is $800 \mathrm{~m}^{3} \mathrm{ha}^{-1}$ for each intervention. For both of the FAO classes, the harvesting of the maize silage occurs in September with self-propelled machines. After transport to the biogas plant, the biomass is stored in silos, and ensilage is executed for wheat biomass. 


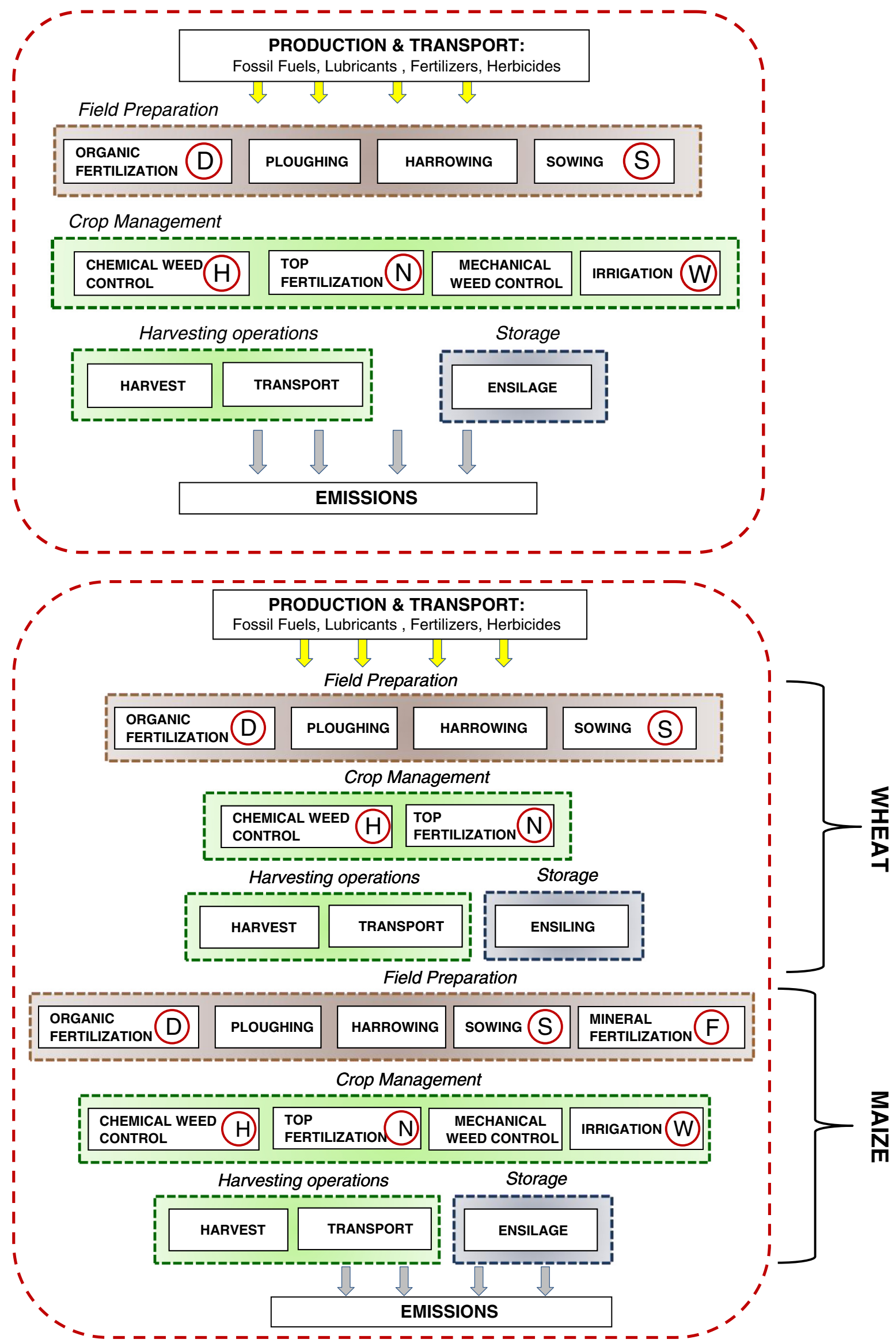

Fig. 1. System boundaries: single crop on the top, double crop on the bottom. Note: $\mathrm{D}=$ digestate, $\mathrm{S}=$ seeds, $\mathrm{H}=$ herbicide, $\mathrm{N}=$ nitrogen fertilizer, $\mathrm{W}=$ water, $\mathrm{F}=$ phosphorous and potassium fertilizers. 
Table 1

Methane potential production for the different crops

\begin{tabular}{llr}
\hline Crop system & Crop & \multicolumn{1}{c}{ Average value } \\
& & \multicolumn{1}{c}{$\mathrm{m}_{\mathrm{N}} / \mathrm{t}_{\mathrm{wb}}$} \\
\hline Single crop & Maize 700 & $102.3 \pm 10.9$ \\
Double crop & Maize 500 & $105.6 \pm 11.9$ \\
& Wheat & $83.1 \pm 11.8$ \\
\hline
\end{tabular}

Large differences exist in the biomass yield and dry matter content between the two different classes of maize: The maize 700 gives a yield that is higher $\left(75 \mathrm{t}_{\mathrm{wb}}\right.$ ha ${ }^{-1}$ with a dry matter content of $\left.34 \%\right)$ than that of maize 500 (48.75 $\mathrm{t}_{\mathrm{wb}} \mathrm{ha}^{-1}$ with a dry matter content of $38 \%)$.

\subsubsection{Wheat}

Together with barley and triticale, wheat is one of the most widespread winter crops in Northern Italy [approximately 340,000 ha in 2011] (Casati, 2011). It can be used for human and animal feeding (grain or fodder) and as biomass for AD plants. When employed as an energy crop, wheat is harvested before the grain ripening, and all of the biomass is harvested and chopped in order to be subsequently ensiled.

Before sowing, the soil is first fertilized with digestate $\left(40 \mathrm{t} \mathrm{ha}{ }^{-1}\right)$. after that, the soil is ploughed and harrowed. The digestate comes from nearby AD plants to which the produced biomass is delivered. The spread digestate, which has a dry matter content of $5.4 \%$, is applied during the second half of September, when the average maximum temperature is $26.5{ }^{\circ} \mathrm{C}$ and the average minimum temperature is $16.2{ }^{\circ} \mathrm{C}$. No rainfall (1 $\mathrm{mm}$ ) occurred during the days following the digestate application. The sowing was performed in October using 35,000 seeds ha ${ }^{-1}$ (200 $\mathrm{kg} \mathrm{ha}^{-1}$ ) in order to obtain a final density of 300-350 plants $\mathrm{m}^{-2}$. First, chemical weed control is carried out following the seeding (preemergency) by spraying terbutilazine and alachlor $\left(5 \mathrm{~kg} \mathrm{ha}^{-1}\right)$. In addition to organic fertilization, chemical fertilizers are also applied in two steps. The first is carried out in November using ammonium nitrate at a rate of $60 \mathrm{~kg} \mathrm{ha}^{-1}$; the second is carried out in February with urea $\left(60 \mathrm{~kg} \mathrm{ha}^{-1}\right)$. However, mechanical weed control and irrigation are not carried out. In May, the cariosside (seed) reaches the waxy ripeness, and the whole crop (straw and grain) is harvested and chopped using a self-propelled forager that simultaneously loads the biomass into farm trailers that are coupled with tractors and driven beside the foragers. The biomass yield is $38.35 \mathrm{t}_{\mathrm{wb}}$ ha ${ }^{1}$ (with a dry matter content of $32 \%)$.

The biomass is transported to the biogas plant (distance $=2.5 \mathrm{~km}$ ). The chopped biomass is ensilaged and stored in horizontal silos for feeding the digesters during the entire year. The ensiling operations are carried out by means of wheeled tractors that are equipped with a frontal loader that compacts and presses the chopped biomass inside the silos.

\subsection{Functional unit}

The functional unit (FU) selected in many LCA studies of energy crops is the mass of biomass (Dressler et al., 2012; Goglio et al., 2012; González-García et al., 2012a,b). However, the biomasses produced from the energy crops assessed have different characteristics and, consequently, different biogas specific productions. Therefore, the mass of biomass does not appear to be the most appropriate FU because it does not allow a fair comparison among the assessed cereals and crop systems.

Considering that the analysis was performed on crops that were specifically cultivated for energy production by means of AD plants, the selected FU was 1 normal cubic metre $\left(1 \mathrm{~m}^{3} \mathrm{~N}\right)$ of potential methane $\left(\mathrm{CH}_{4}\right)$. Methane potential production data were obtained through experimental tests (Table 1) (described in Section 2.5).

For both crop systems, the annual methane potential production was considered by calculating the volume of potential methane obtained from:

1. 1 ha cultivated with maize 700 (year 2011), and

2. 1 ha cultivated with maize 500 and wheat (year 2011).

Table 2

Field and ensilage operations for single crop (maize 700).

\begin{tabular}{|c|c|c|c|c|c|c|c|}
\hline \multirow[t]{2}{*}{ Operation } & \multirow[t]{2}{*}{ NN. } & \multirow[t]{2}{*}{ Month } & \multirow{2}{*}{$\begin{array}{l}\text { Tractor } \\
\text { Mass } \\
\text { Power }\end{array}$} & \multicolumn{3}{|l|}{ Operative machine } & \multirow[t]{2}{*}{ Note } \\
\hline & & & & $\begin{array}{l}\text { Type } \\
\text { Size }\end{array}$ & Mass (kg) & Time (h/ha) & \\
\hline Pre-seeding organic fertilization & 1 & May & $\begin{array}{l}5050 \mathrm{~kg} \\
90 \mathrm{~kW}\end{array}$ & $\begin{array}{l}\text { Manure spreader } \\
20 \mathrm{~m}^{3}\end{array}$ & 2000 & 3.33 & $\begin{array}{l}85 \mathrm{t}_{\mathrm{wb}} \mathrm{ha}^{-1} \\
\text { Digestate }^{[\mathrm{a}]}\end{array}$ \\
\hline Ploughing & 1 & May & $\begin{array}{l}10,500 \mathrm{~kg} \\
190 \mathrm{~kW}\end{array}$ & Plough & 2000 & 1.11 & - \\
\hline Harrowing & 1 & May & $\begin{array}{l}7300 \mathrm{~kg} \\
130 \mathrm{~kW}\end{array}$ & $\begin{array}{l}\text { Rotary Harrow } \\
4.0 \mathrm{~m}\end{array}$ & 1800 & 1.20 & - \\
\hline Sowing & 1 & May & $\begin{array}{l}5050 \mathrm{~kg} \\
90 \mathrm{~kW}\end{array}$ & $\begin{array}{l}\text { Pneumatic seeder } \\
4 \text { lines }\end{array}$ & 900 & 1.00 & $20 \mathrm{~kg} \mathrm{ha}^{-1}$ \\
\hline Chemical weeding & 3 & $\begin{array}{l}\text { May Jun } \\
\text { Jun }\end{array}$ & $\begin{array}{l}4450 \mathrm{~kg} \\
80 \mathrm{~kW}\end{array}$ & $\begin{array}{l}\text { Sprayer } \\
15 \mathrm{~m}\end{array}$ & 600 & 0.33 & $\begin{array}{l}4 \mathrm{~kg} \mathrm{ha}^{-1} \text { lumax } \\
1 \mathrm{~kg} \mathrm{ha}^{-1} \text { dual } \\
1 \mathrm{~kg} \mathrm{ha}^{-1} \text { dual }\end{array}$ \\
\hline Irrigation & 5 & $\begin{array}{l}\text { Jun } \\
\text { Jul } \\
\text { Aug }\end{array}$ & $\begin{array}{l}4450 \mathrm{~kg} \\
80 \mathrm{~kW}\end{array}$ & $\begin{array}{l}\text { Pump } \\
950 \mathrm{~m}^{3} / \mathrm{h}\end{array}$ & 550 & 1.20 & $4400 \mathrm{~m}^{3} \mathrm{ha}^{-1}$ \\
\hline Mechanical weeding & 1 & Jun & $\begin{array}{l}5050 \mathrm{~kg} \\
90 \mathrm{~kW}\end{array}$ & $\begin{array}{l}\text { Weeder } \\
2.8 \mathrm{~m}\end{array}$ & 550 & 0.33 & - \\
\hline Top fertilization & 1 & Jun & $\begin{array}{l}6850 \mathrm{~kg} \\
120 \mathrm{~kW}\end{array}$ & $\begin{array}{l}\text { Fertilizer spreader } \\
2500 \mathrm{dm}^{3}\end{array}$ & 500 & 0.13 & $60 \mathrm{~kg} \mathrm{ha}^{-1}$ urea \\
\hline Harvesting & 1 & Sep & - & $\begin{array}{l}\text { Forage harvester } \\
335 \mathrm{~kW}\end{array}$ & 13,000 & 1.00 & \\
\hline Transport & 1 & Sep & $\begin{array}{l}5050 \mathrm{~kg} \\
90 \mathrm{~kW}\end{array}$ & $\begin{array}{l}3 \text { Farm trailers } \\
30 \mathrm{~m}^{3}\end{array}$ & 5500 & 3.03 & - \\
\hline Ensilage & 1 & Set & $\begin{array}{l}5050 \mathrm{~kg} \\
90 \mathrm{~kW}\end{array}$ & $\begin{array}{l}2 \text { Frontal loader } \\
2 \mathrm{~m}^{3}\end{array}$ & 450 & 3.03 & \\
\hline
\end{tabular}

\footnotetext{
[a] Average composition: $\mathrm{N}=0.40 \% ; \mathrm{P}_{2} \mathrm{O}_{5}=0.08 \%, \mathrm{~K}_{2} \mathrm{O}=0.31 \%$.
} 


\subsection{System boundaries}

The studied system (Fig. 1) included crop cultivation and harvesting, biomass transport, and ensilage to the biogas plant. For the two crop systems, the life cycle of each agricultural process was included within the system boundaries. This life cycle considers raw materials extraction (e.g. fossil fuels and minerals), manufacture (e.g. seeds, fertilizers, chemicals and agricultural machines) and use (diesel fuel consumption and derived combustion). The agronomic inputs for the two crop systems under assessment are shown in Tables 2 and 3, where the characteristics of agricultural machines commonly used for these crops are summarized.

Three different scenarios were considered:

1. The baseline scenario (BS) represents the situation as it was recorded and described within Tables 4 and 5; the average value was considered.

2. Alternative scenario 1 (AS1), or "hypothetical future scenario," is a scenario in which an increase of $15 \%$ of biomass yield (and, subsequently, of $\mathrm{CH} 4$ production) and fertilizer application was assumed. This scenario considers favorable climatic conditions in addition to proper plant nutrition as well as the development of improved maize hybrids (for example, genetically modified organisms with resistance to pests and/or to drought). The impact of yield increase on environmental performances has already been evaluated in several LCA studies (for example. González-García et al., 2012a,b). Although several authors have studied the possibility of getting a yield raise as a consequence of an increase in fertilizer application (Bélanger et al., 2012; El-Fouly et al., 2012; Gagnon et al., 2012; Latković et al., 2012; Tremblay et al., 2012) the increase of fertilizer application has been hypothesized only in order to keep balanced the ratio between nitrogen application and nitrogen removal.

3. Alternative scenario 2 (AS2), or "worst case scenario," is a scenario in which all agricultural inputs were kept constant, and a decrease in yield of $15 \%$ was hypothesized as a consequence of adverse weather conditions (for example, hailstorms, strong drought) or due to inadequate phytosanitary management (for example, unexpected/late detection of Ostrinia nubilalis and or Diabrotica virgifera virgifera attacks).

\subsection{Data collection}

Data (from 2011) concerning field operations, ensilage, and transport were directly obtained via questionnaires that were administered to farmers and via surveys on the field. The farmer provided all

Table 3

Field and ensilage operations for double cropping (wheat + maize 500).

\begin{tabular}{|c|c|c|c|c|c|c|c|c|}
\hline & \multirow[t]{2}{*}{ Operation } & \multirow[t]{2}{*}{ NN. } & \multirow[t]{2}{*}{ Month } & \multirow{2}{*}{$\begin{array}{l}\text { Tractor } \\
\text { Mass } \\
\text { Power }\end{array}$} & \multicolumn{3}{|l|}{ Operative machine } & \multirow[t]{2}{*}{ Note } \\
\hline & & & & & Type Size & Mass (kg) & Time (h/ha) & \\
\hline \multirow[t]{9}{*}{ WHEAT } & Pre-seeding organic fertilization & 1 & Sep & $\begin{array}{l}5050 \mathrm{~kg} \\
90 \mathrm{~kW}\end{array}$ & $\frac{\text { Manure spreader }}{20 \mathrm{~m}^{3}}$ & 2000 & 3.33 & $\begin{array}{l}40 \mathrm{t}_{\mathrm{wb}} \mathrm{ha}^{-1} \\
\text { Digestate }^{[\mathrm{a}]}\end{array}$ \\
\hline & Ploughing & 1 & Sep & $\begin{array}{l}10,500 \mathrm{~kg} \\
190 \mathrm{~kW}\end{array}$ & $\begin{array}{l}\text { Plough } \\
\text { 3-shovel }\end{array}$ & 2000 & 1.11 & \\
\hline & Harrowing & 1 & Sep & $\begin{array}{l}7300 \mathrm{~kg} \\
130 \mathrm{~kW}\end{array}$ & $\begin{array}{l}\text { Rotary harrow } \\
4.0 \mathrm{~m}\end{array}$ & 1800 & 1.20 & \\
\hline & Seeding & 1 & Oct & $\begin{array}{l}5050 \mathrm{~kg} \\
90 \mathrm{~kW}\end{array}$ & Seeder & 900 & 1.00 & $200 \mathrm{~kg} \mathrm{ha}^{-1}$ \\
\hline & Mechanical Weeding & 1 & Oct & $\begin{array}{l}4450 \mathrm{~kg} \\
80 \mathrm{~kW}\end{array}$ & $\begin{array}{l}\text { Spraying } \\
15 \mathrm{~m}\end{array}$ & 600 & 0.33 & $\begin{array}{l}\text { Terbutilazina }+ \\
\text { Alachlor } 5 \mathrm{~kg} \mathrm{ha}^{-1}\end{array}$ \\
\hline & Top fertilization & 2 & $\begin{array}{l}\text { Nov } \\
\text { Feb }\end{array}$ & $\begin{array}{l}6850 \mathrm{~kg} \\
120 \mathrm{~kW}\end{array}$ & $\begin{array}{l}\text { Fertilizer spreader } \\
2500 \mathrm{dm}^{3}\end{array}$ & 500 & 0.13 & $\begin{array}{l}60 \mathrm{~kg} \mathrm{ha}^{-1} \text { ammonium } \\
\text { nitrate } \\
60 \mathrm{~kg} \mathrm{ha}^{-1} \text { urea }\end{array}$ \\
\hline & Harvesting & 1 & May & - & $\begin{array}{l}\text { Forage harvester } \\
335 \mathrm{~kW}\end{array}$ & 13,000 & 1.00 & \\
\hline & Transport & 1 & May & $\begin{array}{l}5050 \mathrm{~kg} \\
90 \mathrm{~kW}\end{array}$ & $\begin{array}{l}2 \text { Farm trailers } \\
30 \mathrm{~m}^{3}\end{array}$ & 5500 & 2.00 & \\
\hline & Ensilage & 1 & May & $\begin{array}{l}5050 \mathrm{~kg} \\
90 \mathrm{~kW}\end{array}$ & $\begin{array}{l}2 \text { Frontal loader } \\
2 \mathrm{~m}^{3}\end{array}$ & 450 & 2.00 & \\
\hline \multirow[t]{12}{*}{ MAIZE 500} & Pre-seeding organic fertilization & 1 & May & $\begin{array}{l}5050 \mathrm{~kg} \\
90 \mathrm{~kW}\end{array}$ & $\begin{array}{l}\text { Manure spreader } \\
20 \mathrm{~m}^{3}\end{array}$ & 2000 & 3.33 & $\begin{array}{l}45 \text { t ha }^{-1} \\
\text { Digestate }\end{array}$ \\
\hline & Ploughing & 1 & May & $\begin{array}{l}10,500 \mathrm{~kg} \\
190 \mathrm{~kW}\end{array}$ & $\begin{array}{l}\text { Plough } \\
\text { 3-shovel }\end{array}$ & 2000 & 1.11 & - \\
\hline & Post-seeding mineral fertilization & 1 & May & $\begin{array}{l}6850 \mathrm{~kg} \\
120 \mathrm{~kW}\end{array}$ & $\begin{array}{l}\text { Fertilizer spreader } \\
2500 \mathrm{dm}^{3}\end{array}$ & 500 & 0.13 & $\begin{array}{l}100 \mathrm{~kg} \mathrm{ha}^{-1} \\
\mathrm{P}_{2} \mathrm{O}_{5} \text { and } \mathrm{K}_{2} \mathrm{O}\end{array}$ \\
\hline & Harrowing & 1 & May & $\begin{array}{l}7300 \mathrm{~kg} \\
130 \mathrm{~kW}\end{array}$ & $\begin{array}{l}\text { Rotary harrow } \\
4,0 \mathrm{~m}\end{array}$ & 1800 & 1.20 & - \\
\hline & Seeding & 1 & May & $\begin{array}{l}5050 \mathrm{~kg} \\
90 \mathrm{~kW}\end{array}$ & $\begin{array}{l}\text { Pneumatic seeder } \\
4 \text { lines }\end{array}$ & 900 & 1.00 & $19 \mathrm{~kg} \mathrm{ha}^{-1}$ \\
\hline & Chemical weeding & 3 & $\begin{array}{l}\text { May Jun } \\
\text { Jun }\end{array}$ & $\begin{array}{l}4450 \mathrm{~kg} \\
80 \mathrm{~kW}\end{array}$ & $\begin{array}{l}\text { Sprayer } \\
15 \mathrm{~m}\end{array}$ & 600 & 0.33 & $\begin{array}{l}1 \mathrm{~kg} \mathrm{ha}^{-1} \text { dual } \\
4 \mathrm{~kg} \mathrm{ha}^{-1} \text { lumax }\end{array}$ \\
\hline & Irrigation & 4 & $\begin{array}{l}\text { Jun, } \\
2 \text { Jul } \\
\text { Aug }\end{array}$ & $\begin{array}{l}4450 \mathrm{~kg} \\
80 \mathrm{~kW}\end{array}$ & $\begin{array}{l}\text { Pump } \\
950 \mathrm{~m}^{3} / \mathrm{h}\end{array}$ & 550 & 1.20 & $3600 \mathrm{~m}^{3} \mathrm{ha}^{-1}$ \\
\hline & Weeding & 1 & Jun & $\begin{array}{l}5050 \mathrm{~kg} \\
90 \mathrm{~kW}\end{array}$ & $\begin{array}{l}\text { Weeder } \\
2.8 \mathrm{~m}\end{array}$ & 550 & 0.33 & \\
\hline & Top fertilization & 1 & Jun & $\begin{array}{l}6850 \mathrm{~kg} \\
120 \mathrm{~kW}\end{array}$ & $\begin{array}{l}\text { Fertilizer spreader } \\
2500 \mathrm{dm}^{3}\end{array}$ & 500 & 0.13 & $60 \mathrm{~kg} \mathrm{ha}^{-1}$ urea \\
\hline & Harvesting & 1 & Sep & - & $\begin{array}{l}\text { Forage Harvester } \\
335 \mathrm{~kW}\end{array}$ & 13,000 & 1.00 & \\
\hline & Transport & 1 & Sep & $\begin{array}{l}5050 \mathrm{~kg} \\
90 \mathrm{~kW}\end{array}$ & $\begin{array}{l}3 \text { Farm trailers } \\
30 \mathrm{~m}^{3}\end{array}$ & 5500 & 3.03 & \\
\hline & Ensilage & 1 & May & $\begin{array}{l}5050 \mathrm{~kg} \\
90 \mathrm{~kW}\end{array}$ & $\begin{array}{l}2 \text { Frontal loader } \\
2 \mathrm{~m}^{3}\end{array}$ & 450 & 3.03 & \\
\hline
\end{tabular}

\footnotetext{
[a] Average composition: $\mathrm{N}=0.40 \% ; \mathrm{P}_{2} \mathrm{O}_{5}=0.08 \%, \mathrm{~K}_{2} \mathrm{O}=0.31 \%$.
} 
Table 4

Data inventory for single crop (data related to $\mathrm{FU}: 1 \mathrm{~m}^{3}{ }_{\mathrm{N}}$ of potential $\mathrm{CH}_{4}$ ).

\begin{tabular}{|c|c|c|}
\hline Input from the technosphere & Units & \\
\hline \multicolumn{3}{|l|}{ Fertilizers } \\
\hline Digestate $\mathrm{e}^{[\mathrm{a}]}$ & $\mathrm{kg}$ & 10.8 \\
\hline Urea as $\mathrm{N}$ & $\mathrm{g}$ & 3.51 \\
\hline \multicolumn{3}{|l|}{ Pesticides } \\
\hline Metolachlor S & $\mathrm{mg}$ & 412.9 \\
\hline Triazine compounds & $\mathrm{mg}$ & 95.29 \\
\hline Mesotrione & $\mathrm{mg}$ & 19.06 \\
\hline \multicolumn{3}{|l|}{ Fuel } \\
\hline Diesel fuel for field operations and transport & $\mathrm{g}$ & 26.4 \\
\hline Diesel fuel for ensilage & $\mathrm{g}$ & 4.53 \\
\hline \multicolumn{3}{|l|}{ Other inputs } \\
\hline Lubricants & $\mathrm{g}$ & 0.63 \\
\hline Maize seeds & $\mathrm{g}$ & 2.54 \\
\hline \multicolumn{3}{|l|}{ Input from the environment } \\
\hline Land use & $\mathrm{m}^{2}$ & 1.27 \\
\hline Irrigation water & $\mathrm{m}^{3}$ & 0.56 \\
\hline \multicolumn{3}{|l|}{ Output to the environment } \\
\hline \multicolumn{3}{|l|}{ Product } \\
\hline Maize 700 silage $^{[\mathrm{b}]}$ & $\mathrm{kg}$ & 9.78 \\
\hline \multicolumn{3}{|l|}{ Emissions to the atmosphere } \\
\hline Ammonia (fertilizer) & $\mathrm{g}$ & 7.33 \\
\hline Nitrous oxide (fertilizer) & $\mathrm{g}$ & 0.49 \\
\hline HC (diesel) & $\mathrm{mg}$ & 64.8 \\
\hline CO (diesel) & $\mathrm{g}$ & 0.34 \\
\hline $\mathrm{CO}_{2}$ (diesel) & $\mathrm{g}$ & 87.9 \\
\hline $\mathrm{NO}_{\mathrm{x}}($ diesel $)$ & $\mathrm{g}$ & 0.69 \\
\hline Particulate (diesel) & $\mathrm{mg}$ & 44.06 \\
\hline Metolachlor S & $\mathrm{mg}$ & 9.62 \\
\hline Mesotrione & $\mathrm{mg}$ & 0.01 \\
\hline Triazine compounds & $\mathrm{mg}$ & 0.10 \\
\hline \multicolumn{3}{|l|}{ Emissions to water } \\
\hline Phosphate & $\mathrm{mg}$ & 86.39 \\
\hline $\mathrm{NO}_{3}$ & $\mathrm{~g}$ & 2.46 \\
\hline Metolachlor S & $\mathrm{mg}$ & 0.36 \\
\hline Mesotrione & $\mathrm{mg}$ & 0.19 \\
\hline Triazine compounds & $\mathrm{mg}$ & 7.34 \\
\hline
\end{tabular}

[a] = By-product of AD: it does not contribute to environmental impact; [b] = Losses ( $5 \%$ of harvested biomass) due to the transport and the ensilage operation were taken into consideration (Bacenetti et al., 2013).

information regarding fertilizer, pesticide (registered in a mandatory document called "Quaderni di campagna"), and water use. The diesel fuel consumption was partly measured (by evaluating the volume of fuel used to fill up fuel tanks to the brim) and partly estimated using the model SE ${ }^{3} \mathrm{~A}$ (Fiala and Bacenetti, 2012).

Emissions due to the fertilizers were included: nitrogen emissions (nitrate, ammonia, and nitrous oxide) were computed following the model proposed by Brentrup et al. (2000), while phosphate emissions were calculated in accordance with Smil (2000). Climatic data for 2011, which were necessary for calculating fertilizer emissions, were obtained from the meteorological station closest to the farm.

Pesticide emissions were also estimated using PestLCI (Birkved and Hauschild, 2006), a model that quantifies the emissions to different environmental compartments (i.e. groundwater, surface water, and air). Regarding fuel use, the emissions that each machine in field operations generated were estimated using data from the Swiss Federal Office for the Environment (Federal Department of the Environment, Transport, Energy and Communications, or DETEC) (DETEC, 2013). Background data for seed production, diesel fuel, fertilizers, and pesticides were obtained from the Ecoinvent database [version 2.2] (Frischknecht et al., 2007) and the LCA Food DK database (Nielsen et al., 2003).

No change in the overall soil carbon content has been assumed because the fields were previously dedicated to cereal cultivation
Table 5

Data inventory for double crop (data related to $\mathrm{FU}: 1 \mathrm{~m}^{3}{ }_{\mathrm{N}}$ of potential $\mathrm{CH}_{4}$ ).

\begin{tabular}{|c|c|c|}
\hline Input from the technosphere & Units & \\
\hline \multicolumn{3}{|l|}{ Fertilizers } \\
\hline Digestate $^{[a]}$ & $\mathrm{kg}$ & 10.73 \\
\hline Urea as $\mathrm{N}$ & $\mathrm{g}$ & 6.97 \\
\hline Fertilizer $\mathrm{P}_{2} \mathrm{O}_{5}$ & $\mathrm{~g}$ & 12.63 \\
\hline Fertilizer $\mathrm{K}_{2} \mathrm{O}$ & $\mathrm{g}$ & 12.63 \\
\hline \multicolumn{3}{|l|}{ Pesticides } \\
\hline Metolachlor S & mg & 284.15 \\
\hline MCPA & mg & 101.03 \\
\hline Triazine compounds & $\mathrm{mg}$ & 94.72 \\
\hline Clopyralid & $\mathrm{mg}$ & 10.10 \\
\hline Fluroxypyr & $\mathrm{mg}$ & 20.21 \\
\hline Mesotrione & $\mathrm{mg}$ & 18.94 \\
\hline \multicolumn{3}{|l|}{ Fuel } \\
\hline Diesel fuel for field operations \& transport & $\mathrm{g}$ & 38.07 \\
\hline Diesel fuel for ensilage & $\mathrm{g}$ & 4.84 \\
\hline \multicolumn{3}{|l|}{ Other inputs } \\
\hline Lubricants & $\mathrm{g}$ & 0.88 \\
\hline Maize seeds & $\mathrm{g}$ & 2.40 \\
\hline Wheat seeds & $\mathrm{g}$ & 25.26 \\
\hline \multicolumn{3}{|l|}{ Input from the environment } \\
\hline Land use & $\mathrm{m}^{2}$ & 1.26 \\
\hline Irrigation water & $\mathrm{m}^{3}$ & 0.45 \\
\hline \multicolumn{3}{|l|}{ Output to the environment } \\
\hline \multicolumn{3}{|l|}{ Products } \\
\hline Maize 500 silage $^{[\mathrm{b}]}$ & $\mathrm{kg}$ & 5.87 \\
\hline Wheat silage & $\mathrm{kg}$ & 4.57 \\
\hline \multicolumn{3}{|l|}{ Emissions to the atmosphere } \\
\hline Ammonia (fertilizer) & $\mathrm{g}$ & 7.82 \\
\hline Nitrous oxide (fertilizer) & $\mathrm{g}$ & 0.56 \\
\hline HC (diesel) & $\mathrm{mg}$ & 90.84 \\
\hline CO (diesel) & $\mathrm{g}$ & 0.48 \\
\hline $\mathrm{CO}_{2}$ (diesel) & $\mathrm{g}$ & 124.85 \\
\hline $\mathrm{NO}_{\mathrm{x}}($ diesel $)$ & $\mathrm{g}$ & 0.97 \\
\hline Particulate (diesel) & $\mathrm{mg}$ & 69.02 \\
\hline Metolachlor S & mg & 6.91 \\
\hline Mesotrione & $\mathrm{mg}$ & 0.01 \\
\hline MCPA & $\mathrm{mg}$ & 3.84 \\
\hline Triazine compounds & $\mathrm{mg}$ & 0.10 \\
\hline Clopyralid & $\mu g$ & 23.24 \\
\hline Fluroxypyr & $\mathrm{mg}$ & 0.36 \\
\hline \multicolumn{3}{|l|}{ Emissions to water } \\
\hline Phosphate & $\mathrm{mg}$ & 212.2 \\
\hline Metolachlor S & $\mathrm{mg}$ & 0.26 \\
\hline МСРА & mg & 2.77 \\
\hline Mesotrione & $\mathrm{mg}$ & 0.18 \\
\hline Triazine compounds & $\mathrm{mg}$ & 6.63 \\
\hline Clopyralid & $\mathrm{mg}$ & 0.31 \\
\hline Fluroxypyr & $\mathrm{mg}$ & 44.66 \\
\hline
\end{tabular}

[a] = By-product of AD: it does not contribute to environmental impact; [b] = Losses (5\% of harvested biomass) due to the transport and the ensilage operation were taken into consideration (Bacenetti et al., 2013).

(González-García et al., 2012a,b). Tables 4 and 5 report data inventories for the single and double crop under study.

Data concerning $\mathrm{CH}_{4}$ potential production were obtained by means of laboratory experimental tests. For the different biomasses, the specific productions were obtained using lab-scale AD tests; unstirred ladfermenters (volume: $2.5 \mathrm{dm}^{3}$ ) were utilized. These were made up of a hermetically sealed glass jar equipped with a metallic cover containing a valve through which the biogas produced reaches the corresponding gasometer. Each gasometer is made from a methacrylate pipe (volume $3.5 \mathrm{dm}^{3}$ ) atop which are fitted two hoses: One transports the biogas from the fermenter, and one, equipped with a valve, can be used for gasometer recharge with the water solution (saturated with $\mathrm{CaCO}_{3}$ to 
Table 6

Evaluated impact categories (CML 2000).

\begin{tabular}{ll}
\hline Impact categories & Unit of measure \\
\hline Ozone layer depletion (ODP) & $\mathrm{kg}$ CFC-11equivalents \\
Human toxicity & 1 -4-dichlorobenzene equivalents \\
Fresh water aquatic ecotoxicity & 1.4-dichlorobenzene equivalents \\
Marine aquatic ecotoxicity & 1.4 -dichlorobenzene equivalents \\
Terrestrial ecotoxicity & 1.4 -dichlorobenzene equivalents \\
Photochemical oxidation & $\mathrm{kg}$ ethylene equivalents \\
Global warming (GWP100) & $\mathrm{kg}$ carbon dioxide equivalents \\
Acidification & $\mathrm{kg} \mathrm{SO}$ equivalents \\
Abiotic depletion & $\mathrm{kg} \mathrm{antimony} \mathrm{equivalents}_{\text {Eutrophication }} \mathrm{kg} \mathrm{PO}_{4}$ equivalents \\
\hline
\end{tabular}

prevent $\mathrm{CO}_{2}$ solubilisation into water) of which it is full at the beginning of the measurement.

Samples of fermenting material from different full-scale AD were collected to be used as inoculum. Before fermenters were set up, they were filtered with $2 \mathrm{~mm}$ sieves and placed at $40{ }^{\circ} \mathrm{C}$ for $48 \mathrm{~h}$ to minimize the amount of inoculum biogas production.

In each fermenter, the inoculum/substrate ratio was kept at 2:1 on a volatile solids basis (Vismara et al., 2008): On average, each fermenter contained $2 \mathrm{~kg}$ of inoculum (total solids $3 \% \pm 0.2$ of raw material) and $30 \mathrm{~g}$ of dried biomass. Before digestion, all substrates were ground. During the experimental tests, the temperature in each fermenter was $40{ }^{\circ} \mathrm{C}$. To keep the biomass conditions as homogeneous as possible

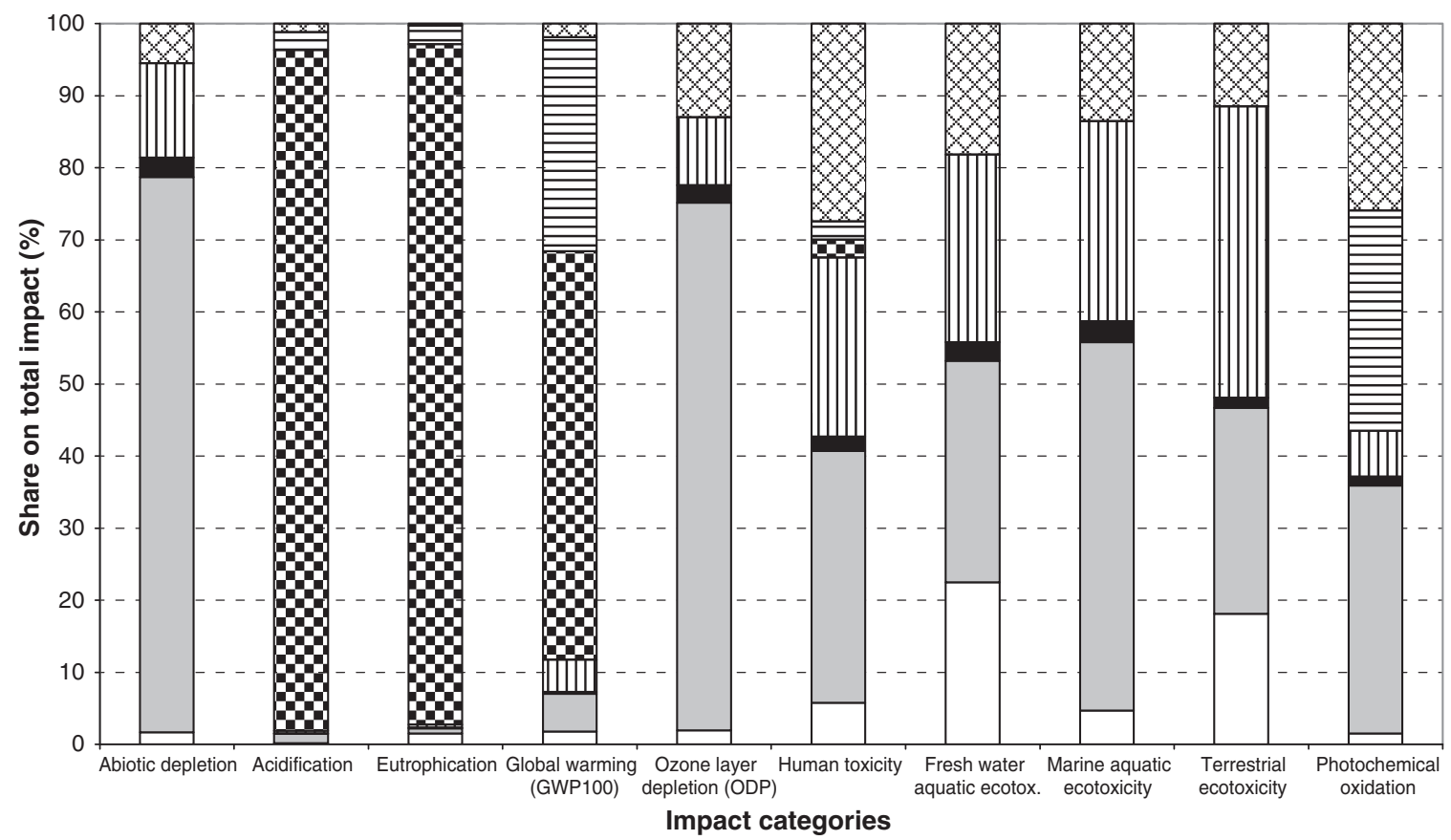

$\square$ Maize seed $\square$ Diesel fuel Lubricants UUrea, as N Fertilizers emissions 日Diesel emissions $\square$ Pesticides production

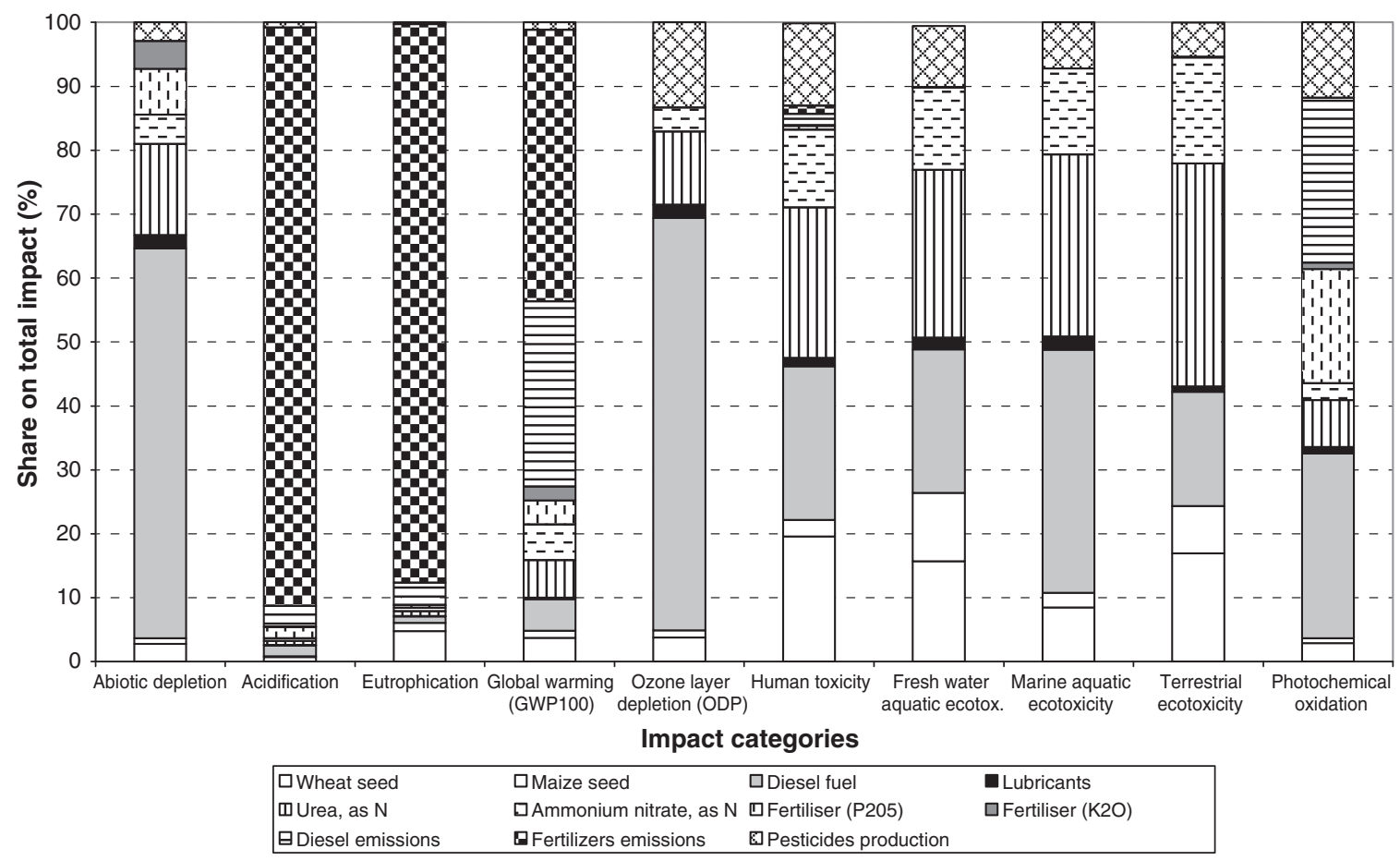

Fig. 2. Environmental impact of $1 \mathrm{~m}^{3}{ }_{\mathrm{N}}$ of $\mathrm{CH}_{4}$ : from maize 700 (top) and from wheat + maize 500 (bottom). 

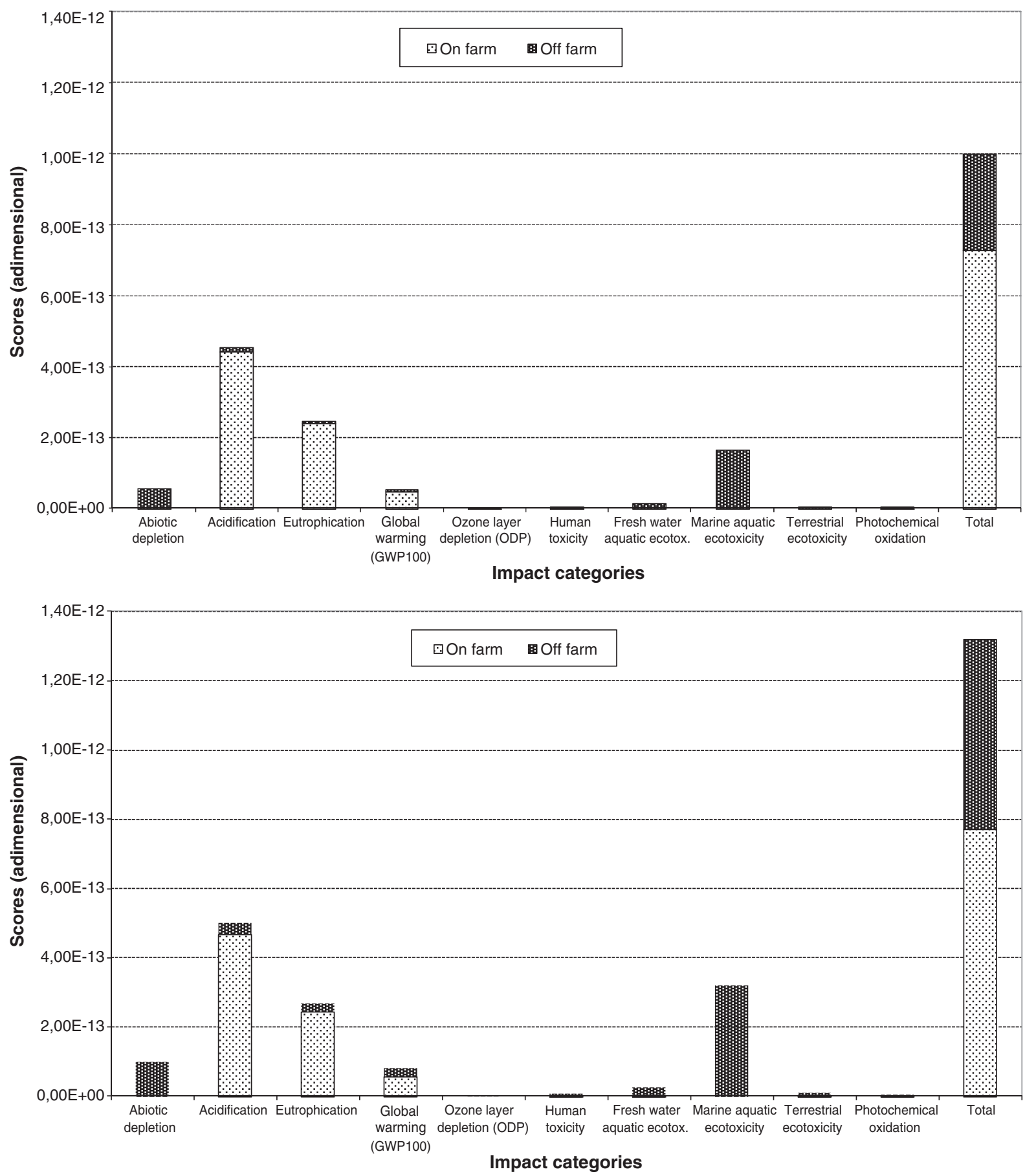

Fig. 3. Environmental impact (subdivided into "on-farm" and "off-farm") to obtain $1 \mathrm{~m}^{3}{ }_{\mathrm{N}}$ of $\mathrm{CH}_{4}$ from the cultivation of maize 700 (top) and of wheat + maize 500 (bottom).

and to facilitate biogas collection, the fermenters were shaken daily. Biogas production was recorded daily by reading the centimeters run in the gasometers and by calculating the equivalent volume.

Before AD, the dry matter total solid (TS) content was determined following a 24-h drying period at $105{ }^{\circ} \mathrm{C}$, while volatile solid (VS) content was determined as a percentage of TS, according to APHA (1998). Biogas composition in terms of $\mathrm{CH}_{4}$ percentage was monitored by means of one "Binder Combigas GA-m" (from Binder, D) portable gas analyzer.

\subsection{Methods}

Among the steps defined within the life cycle impact assessment stage of the standardized LCA methodology, classification, characterization and normalization stages were carried out in this study. The characterization factors reported by the Centre of Environmental Science of Leiden University (CML 2001 method) were used (Guinée et al., 2002). The impact categories evaluated according to the CML method are reported in Table 6. A life cycle impact assessment (LCIA) was performed using SimaPro software (Goedkoop et al., 2008). LCIA was performed for the three different scenarios as previously discussed.

\section{Results and discussion}

The environmental impact linked to $\mathrm{CH}_{4}$ production from maize 700 is widely determined, as expected, by maize cultivation. Ensilage plays a secondary role in the environmental burden for all of the three crops 
Table 7

Comparison between $1 \mathrm{~m}^{3} \mathrm{~N}$ obtained from maize 700 (single crop) and from wheat + maize500 (double crop). The worst crop system was set at 100 .

\begin{tabular}{|c|c|c|}
\hline \multirow[t]{2}{*}{ Impact categories } & Single crop & Double crop \\
\hline & $\%$ & $\%$ \\
\hline Abiotic depletion & 57.70 & 100 \\
\hline Acidification & 90.70 & 100 \\
\hline Eutrophication & 91.83 & 100 \\
\hline Global warming (GWP100) & 66.94 & 100 \\
\hline Ozone layer depletion (ODP) & 63.59 & 100 \\
\hline Human toxicity & 49.13 & 100 \\
\hline Fresh water aquatic ecotox. & 51.80 & 100 \\
\hline Marine aquatic ecotoxicity & 53.51 & 100 \\
\hline Terrestrial ecotoxicity & 44.46 & 100 \\
\hline Photochemical oxidation & 60.20 & 100 \\
\hline
\end{tabular}

evaluated. Considering its importance in terms of environmental impact, the cultivation phase is the stage of the life cycle that is worth attention. Fig. 2 shows, for the two crop systems, the contributions to the impact categories of each input and output for obtaining $1 \mathrm{~m}^{3}{ }_{\mathrm{N}}$ of $\mathrm{CH}_{4}$.

The critical factors are: fertilizer emissions (decisive for acidification, eutrophication and global warming potential), diesel fuel emissions (important for global warming potential and photochemical oxidation), diesel fuel production (decisive for abiotic and ozone layer depletion), pesticide production (important for human toxicity), and urea production. In more detail, for both the crop systems analyzed, approximately $90 \%$ of eutrophication and acidification derived from fertilizer emissions. Global warming potential originating from fertilizer emissions was over $50 \%$ for the single crop and $40 \%$ for the double crop. The abiotic and ozone layer depletion impacts are primarily caused by diesel fuel consumption in both cases. Diesel fuel emissions are responsible for almost $30 \%$ of GWP and photochemical oxidation impacts. The contribution of urea production ranged from 25\% (human and water toxicity) to 30\% (double crop) and 40\% (single crop) (terrestrial ecotoxicity). Pesticides production was relevant primarily for the single crop; its contribution ranged from $12 \%$ (terrestrial ecotoxicity) to almost 30\% (human toxicity).

Normalized data ${ }^{1}$, shown in Fig. 3, were subdivided into "on-farm" and "off-farm" impacts. On-farm impacts represent the environmental burden derived directly from farm activities (such as diesel fuel emissions and fertilizers emissions); meanwhile, off-farm impacts are not directly related to farm activities (inputs production).

For the single crop system, the overall environmental burden mainly stems from on-farm impacts. For the double crop, the overall burden is almost equally due to both on-farm and off-farm impacts. For both the single and double crop, the most relevant impact categories were acidification, eutrophication, marine aquatic ecotoxicity and abiotic depletion. The first two were mainly caused by farm activities, the latter two were the result of off-farm activities.

Comparing the cultivation phases for the single (maize 700) and double (maize $500+$ wheat) crop, it can be stated that for each impact category, the cultivation of maize 700 (to obtain $1 \mathrm{~m}^{3}$ of methane) in the single crop system is environmentally more sustainable than it is in the double crop system.

The comparison in terms of the environmental impact of $1 \mathrm{~m}^{3} \mathrm{~N}$ of $\mathrm{CH}_{4}$ obtained from maize 700 and from maize 500 plus wheat turns out to be very favourable for maize 700 (Table 7). The environmental burden of the double crop is greater than that of the single crop for each impact category; for acidification and eutrophication, the differences are less evident $(<10 \%)$. Knowing that fertilizer emissions largely

\footnotetext{
${ }^{1}$ The CML method includes data normalization: the results for each impact category were divided by a reference. This reference is the average inhabitant environmental load (for each impact category) in Europe in 1995. The normalization step allows the obtaining of adimensional scores.
}

Table 8

Values for the impact categories.

\begin{tabular}{|c|c|c|c|}
\hline Impact category & Units & $\begin{array}{l}\text { Methane potential } \\
\text { from maize } 700\end{array}$ & $\begin{array}{l}\text { Methane potential from } \\
\text { maize } 500+\text { wheat }\end{array}$ \\
\hline Abiotic depletion & $\mathrm{kg} \mathrm{Sb} \mathrm{eq}$ & $9.26 \mathrm{E}-04$ & $1.61 \mathrm{E}-03$ \\
\hline Acidification & $\mathrm{kg} \mathrm{SO}_{2} \mathrm{eq}$ & $1.25 \mathrm{E}-02$ & $1.38 \mathrm{E}-02$ \\
\hline Eutrophication & $\mathrm{kg} \mathrm{PO}_{4}$ eq & $3.12 \mathrm{E}-03$ & $3.36 \mathrm{E}-03$ \\
\hline $\begin{array}{l}\text { Global warming } \\
\text { (GWP100) }\end{array}$ & $\mathrm{kg} \mathrm{CO}$ eq & $2.71 \mathrm{E}-01$ & $4.10 \mathrm{E}-01$ \\
\hline $\begin{array}{l}\text { Ozone layer depletion } \\
\text { (ODP) }\end{array}$ & $\mathrm{kg}$ CFC-11 eq & $1.87 \mathrm{E}-08$ & $2.95 \mathrm{E}-08$ \\
\hline Human toxicity & kg 1.4-DB eq & $3.14 \mathrm{E}-02$ & $6.26 \mathrm{E}-02$ \\
\hline $\begin{array}{l}\text { Fresh water aquatic } \\
\text { ecotox. }\end{array}$ & kg 1.4-DB eq & $7.39 E-03$ & $1.43 E-02$ \\
\hline $\begin{array}{c}\text { Marine aquatic } \\
\text { ecotoxicity }\end{array}$ & kg 1.4-DB eq & $2.04 \mathrm{E}+01$ & $3.82 \mathrm{E}+01$ \\
\hline Terrestrial ecotoxicity & kg 1.4-DB eq & $2.10 \mathrm{E}-04$ & $4.73 \mathrm{E}-04$ \\
\hline Photochemical oxidation & $\mathrm{kg} \mathrm{C}_{2} \mathrm{H}_{4}$ eq & $2.91 \mathrm{E}-05$ & $4.84 \mathrm{E}-05$ \\
\hline
\end{tabular}

influence these impact categories, the main reason for such a result is $\mathrm{N}$ leaching. Notwithstanding that for the double crop more fertilizer is used, no leaching occurs (according to a calculation from Brentrup et al., 2000). On the contrary, cultivation of maize 700, although it requires a lower fertilizer application, determines nitrate leaching, which causes acidification and eutrophication. Table 8 shows scores for all impact categories for methane from the single and double crop systems.

Considering the maximal and minimal $\mathrm{CH}_{4}$ production (see Table 1), the overall environmental impact grows as $\mathrm{CH}_{4}$ production decreases. Concerning the comparison among different scenarios (BS, AS1, AS2) the following can be considered. For maize 700 (Fig. 4), the reduction of the yields (AS2) (keeping all other conditions constant except for the diesel fuel used for ensilage) leads to a proportional decrease of $\mathrm{CH}_{4}$ production, causing an aggravation of the environmental burden of approximately the same entity than the yield decrease (15\%). On the contrary, in AS1, all environmental impacts decrease ( $-20 \%$ approximately) except for acidification and eutrophication, which remain essentially the same.

For maize 500 plus wheat (Fig. 4), comparing BS with AS2, the same considerations could be made except that eutrophication varies more than the yield reduction does. In AS2, the lower yield leads to nitrate leaching (absent in BS), which exacerbates the disparity for the eutrophication impact category.

Considering that it is essential that the production of feedstock is carried out under sustainable conditions, in recent years, several LCA studies have been carried out to evaluate the environmental impact of energy crops cultivation (Dressler et al., 2012; Goglio et al., 2012; González-García et al., 2012b; Bachmaier et al., 2013; Ghahderijani et al., 2013). Our results are in agreement with this literature. In fact, although the use of a different FU does not allow for a strict comparison, all these LCA studies highlighted that the process hotspots are: i) nitrogen fertilization (which involves remarkable impacts due to its production as well as its application into the soil); ii) diesel fuel consumption (mainly for ploughing and harvest, field operations with high power requirements); and iii) pesticide utilization for impact categories such as human toxicity and terrestrial ecotoxicity. In more detail, regarding fertilizer emissions Dressler et al. (2012) and González-García et al. (2012b) also reported a strong relation between organic fertilization and eutrophication.

\section{Conclusions}

This study compares the environmental performances of a single and double crop system. The evaluation has been made using $1 \mathrm{~m}^{3}{ }_{\mathrm{N}}$ of methane as a functional unit due to the final use of the crops considered. 


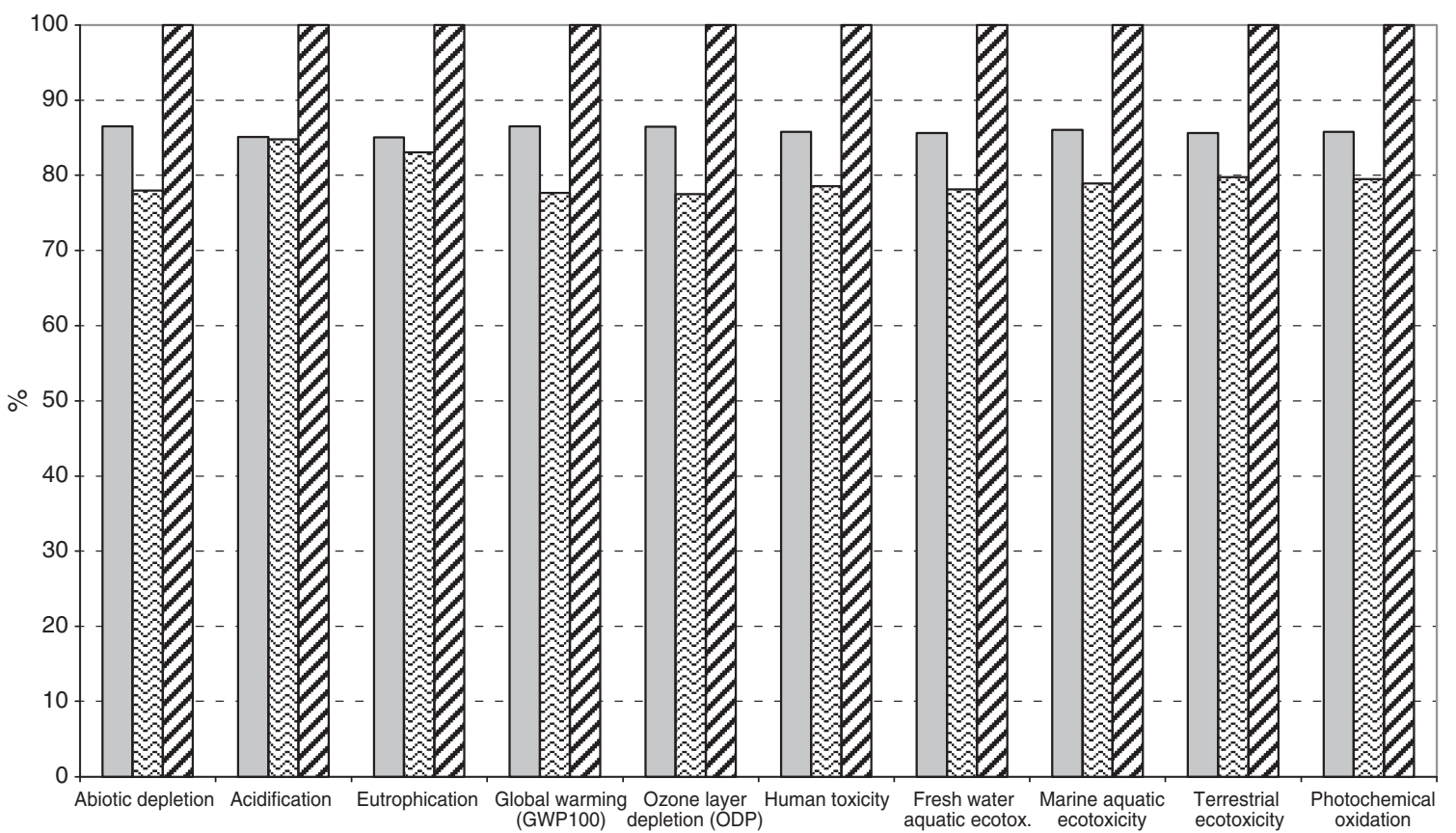

$\square$ Baseline scenario (BS) $\square$ Alternative scenario 1 (SC1) $D$ Alternative scenario 2 (SC2)

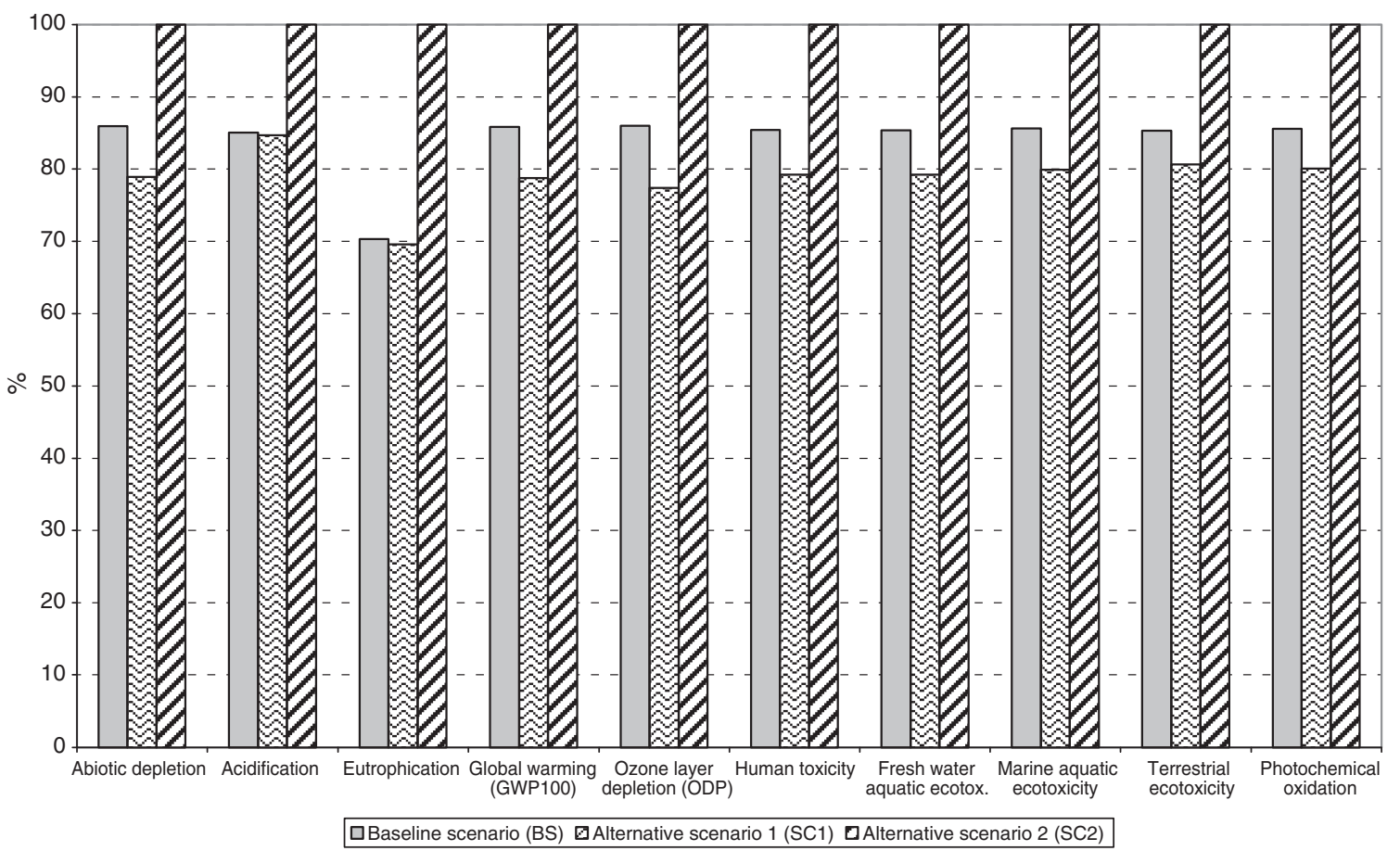

Fig. 4. Comparison among different scenarios for maize 700 (top) and wheat + maize 500 (bottom).

Results from the analysis point out that for all impact categories taken into consideration, the double crop system appears to have the worse environmental performance compared to the single crop system. This means that the greater amount of biomass (silage) obtained with the double crop system is less than proportional to the environmental burden that results from the bigger quantity of inputs needed for the double crop system.

Comparing scenarios that differ for biomass yields, it is evident that the more the yield increases, the more the environmental burden decreases. The same supposition can be drawn for specific methane production: The more the methane specific production increases, the better the environmental performance of the system.

The analysis executed highlighted that the nitrogen cycle and their linked emissions are relevant for the environmental burden of maize and wheat cultivation, especially for some impact categories (namely acidification and eutrophication). Therefore, the model chosen to estimate nitrogen emissions in the environment is a critical factor in this kind of analysis due to its influence on the final result. For this reason, the analysis of this aspect of cultivation should be, if possible, performed with site-specific models. 
Because the biomass produced in the two crop systems under study is used to feed anaerobic digestion plants, the next step of our study will be the analysis of the conversion phase of the biomass into biogas and then into electricity. The results of the current analysis represent the first essential step for the whole life cycle assessment of electricity production from $A D$ realized in agricultural plants.

\section{Acknowledgments}

The present work is funded by Regione Lombardia - Fondo per la Promozione di Accordi Istituzionali, project BIOGESTECA 15083/RCC". In addition, the Authors thank Regione Lombardia which financed a Postdoctoral Research Fellowship ("Progetto Dote Ricerca" financed by FSE - Regione Lombardia).

\section{References}

Angelidaki I, Ellegaard L. Codigestion of manure and organic wastes in centralized biogas plants: status and future trends. Appl Biochem Biotechnol 2003;109: 95-105.

Appels L, Lauwers J, Degrève J, Helsen L, Lievens B, Willems K, et al. Anaerobic digestion in global bio-energy production: potential and research challenges. Renew Sustain Energy Rev 2011;15:4295-301.

Audsley E. Harmonisation of environmental life cycle assessment for agriculture. Final Report, Concerted Action AIR3-CT94-2028. European Commission, DG VI Agriculture; 1997. p. 1-139.

Bacenetti J, González-García S, Mena A, Fiala M. Life cycle assessment: an application to poplar for energy cultivated in Italy. J Agric Eng 2012a;43:72-8.

Bacenetti J, Mena A, Negri M, Cantarella P, Bocchi S, Fiala M. Energetic and environmental balance of a biogas plant in Northern Italy. Proceedings CIGR-AgEng International Conference on Agricultural EngineeringValencia. CIGR-AgEng; 2012b.

Bacenetti J, Negri M, Fiala M, Gonzalez Garcia S. Anaerobic digestion of different feedstock: impact on energetic and environmental balances of biogas process. Sci Total Environ 2013;463-464:541-51.

Bachmaier J, Effenberger M, Gronauer A. Greenhouse gas balance and resource demand of biogas plants in agriculture. Eng Life Sci 2010;10:560-9.

Bachmaier H, Effenberger M, Gronauer A, Boxberger J. Changes in greenhouse gas balance and resource demand of biogas plants in southern Germany after a period of three years. Waste Manag Res 2013;31(4):368-75.

Bélanger G, Claessens A, Ziadi N. Grain N and P relationships in maize. Field Crop Res 2012;126:1-7.

Birkved M, Hauschild M. PestLCl-a model for estimating field emissions of pesticides in agricultural LCA. Ecol Model 2006;198:433-51.

Börjesson P, Berglund M. Environmental systems analysis of biogas systems - Part II: The environmental impact of replacing various reference systems. Biomass Bioenergy 2007;31:326-44.

Börjesson P, Mattiasson B. Biogas as a resource-efficient vehicle fuel. Trends Biotechnol 2008;26:7-13

Brentrup F, Küsters J, Lammel J, Kuhlmann H. Methods to estimate on-field nitrogen emissions from crop production as an input to LCA studies in the agricultural sector. Int J Life Cycle Assess 2000;5:349-57.

Brentrup F, Küsters J, Kuhlmann H, Lammel J. Application of the life cycle assessment methodology to agricultural production: an example of sugar beet production with different forms of nitrogen fertilisers. Eur J Agron 2001;14:221-33.

Brentrup F, Küsters J, Lammel J, Barraclough P, Kuhlmann H. Environmental impact assessment of agricultural production systems using the life cycle assessment (LCA) methodology II. The application to $\mathrm{N}$ fertilizer use in winter wheat production systems. Eur J Agron 2004;20:265-79.

Buratti C, Fantozzi F. Life cycle assessment of biomass production: development of a methodology to improve the environmental indicators and testing with fiber sorghum energy crop. Biomass Bioenergy 2010;34:1513-22.

Capponi S, Fazio S, Barbanti L. $\mathrm{CO}_{2}$ savings affect the break-even distance of feedstock supply and digestate placement in biogas production. Renew Energy 2012:37:45-52.

Carozzi M, Ferrara RM, Rana G, Acutis M. Evaluation of mitigation strategies to reduce ammonia losses from slurry fertilisation on arable lands. Sci Total Environ 2013;449: 126-33.

Casati D. Le superfici possono ricominciare a salire. Terra Vita 2011:35:40-6.

Casati D. Annata davvero difficile urge risalire la china. Terra Vita 2013;6:40-4.

Cherubini F, Bargigli S, Ulgiati S. Life cycle assessment (LCA) of waste management strategies: landfilling, sorting plant and incineration. Energy 2009;34:2116-23.

Clemens J, Trimborn M, Weiland P, Amon B. Mitigation of greenhouse gas emissions by anaerobic digestion of cattle slurry. Agr Ecosyst Environ 2006;112(2-3):171-7.

Del Prado A, Mas K, Pardo G, Gallejones P. Modelling the interactions between C and $\mathrm{N}$ farm balances and GHG emissions from confinement dairy farms in northern Spain. Sci Total Environ 2013. http://dx.doi.org/10.1016/j.scitotenv.2013.03.064 [in press].

DETEC(Federal Department of the Environment, Transport, Energy and Communications). http://www.bafu.admin.ch/index.html?lang=it. [last visit 05/02/2013]
Dressler D, Loewen A, Nelles M. Life cycle assessment of the supply and use of bioenergy: impact of regional factors on biogas production. Int J Life Cycle Assess 2012;17(9): 1104-15.

Edelmann W, Baier U, Engeli H. Environmental aspects of the anaerobic digestion of the organic fraction of municipal solid wastes and of solid agricultural wastes. Water Sci Technol 2005;52:203-8.

El-Fouly M, Abou El-Nour E, Shaaban S, Zeidan M. Effect of different levels of NPK and micronutrients fertilization on yield and nutrient uptake of maize plants. J Am Sci 2012;8(8):209-14

European Parliament Council. Report from the commission to the council and the European Parliament on sustainability requirements for the use of solid and gaseous biomass sources in electricity, heating and cooling; 2010.

European parliament and council. Directive 2009/28/EC on the promotion of the use of energy from renewable sources, vol. L 283. Official Journal of the European Communities; 2009. p. 33

Fazio S, Monti A. Life cycle assessment of different bioenergy production systems including perennial and annual crops. Biomass Bioenergy 2011;35:4868-78.

Fiala M, Bacenetti J. Economic, energetic and environmental impact in Short Rotation Coppice harvesting operations. Biomass Bioenergy 2011;42:107-13.

Fiala M, Bacenetti J. Model for the economic, energetic and environmental evaluation in biomass productions. J Agric Eng 2012;42:26-35.

Finnveden G, Hauschild M, Ekvall T, Guine J, Heijungs R, Hellwege S, et al. Recent developments in Life Cycle Assessment. J Environ Manage 2009;91:1-21.

Frischknecht R, Jungbluth N, Althaus HJ, Doka G, Heck T, Hellweg S, et al. Overview and methodology. Ecoinvent report No. 1. Dübendorf: Swiss Centre for Life Cycle Inventories; 2007.

Gagnon B, Ziadi N, Grant C. Urea fertilizer forms affect grain corn yield and nitrogen use efficiency. Can J Soil Sci 2012;92(2):341-51.

Gasol CM, Gabarrell X, Anton A, Rigola M, Carrasco J, Ciria P, et al. LCA of poplar bioenergy system compared with Brassica carinata energy crop and natural gas in regional scenario. Biomass Bioenergy 2009;33:119-29.

Ghahderijani M, Komleh S, Keyhani A, Sefeedpari P. Energy analysis and life cycle assessment of wheat production in Iran. Afr J Agric Res 2013;8(18):1929-39.

Goedkoop M, de Schryver A, Oele M. Introduction to LCA with SimaPro 7. the Netherlands: PRé Consultants; 2008.

Goglio P, Bonari E, Mazzoncini M. LCA of cropping systems with different external input levels for energetic purposes. Biomass Bioenergy 2012;42:33-42.

González-García S, Bacenetti J, Murphy R, Fiala M. Present and future environmental impact of poplar cultivation in Po valley (Italy) under different crop management systems. J Clean Prod 2012a;26:56-66.

González-García S, Bacenetti J, Negri M, Fiala M, Arroja L. Comparative environmental performance of three different annual energy crops for biogas production in Northern Italy. J Clean Prod 2012b;43:71-83.

González-García S, Mola-Yudego B, Dimitriou I, Aronsson P, Murphy R. Environmental assessment of energy production based on long term commercial willow plantations in Sweden. Sci Total Environ 2012c;421-422:210-9.

Guinée JB, Gorrée M, Heijungs R, Huppes G, Kleijn R, Koning A, et al. Handbook on life cycle assessment. Operational guide to the ISO standards. I: LCA in perspective. Ila: Guide. IIb: Operational annex. III: Scientific background. Kluwer. Dordrecht: Academic Publishers; 2002. p. 692.

IPCC. Agriculture, forestry and other land use. In: Eggleston, et al, editor. IPCC Guidelines for National Greenhouse Gas Inventories, Prepared by the National Greenhouse Gas Inventories Programme; 2006.

ISO. Environmental management-life cycle assessment-principles and framework (ISO 14040). Brussels: European Committee for Standardization; 2006.

ISTAT - Istituto Nazionale di Statistica. Tavola C02 - Superficie (ettari) e produzione (quintali): riso, mais, sorgo, altri cereali. Dettaglio per regione - Anno. http://agri. istat.it/jsp/dwExcel.jsp?q = plC020000010000012000\&an $=2011 \& \mathrm{ig}=1 \& \mathrm{ct}=244 \& \mathrm{id}=$ 18A|15A|25A, 2011. [last access 21/12/12].

Jury C, Benetto E, Koster D, Schmitt B, Welfring J. Life Cycle Assessment of biogas production by monofermentation of energy crops and injection into the natural gas grid. Biomass Bioenergy 2010;34:54-66.

Kimming M, Sundberg C, Nordberg Å, Baky A, Bernesson S, Norén O, et al. Biomass from agriculture in small-scale combined heat and power plants - a comparative life cycle assessment. Biomass Bioenergy 2011;35:1572-81.

Lansche J, Müller J. Life cycle assessment of energy generation of biogas fed combined heat and power plants: environmental impact of different agricultural substrates. Eng Life Sci 2012;12:313-20.

Latković D, Marinković D, Crnobarac J, Jaćimović G, Berenji J, Sikora J. Influence of crop residues and increasing rates of nitrogen on the yield of corn. Res J Agric Sci 2012;44(3):61-6.

Ministero dello Sviluppo Economico. Decreto ministeriale 6 luglio 2012 - Incentivi per energia da fonti rinnovabili elettriche non fotovoltaiche. http://www.sviluppoeconomico.gov.it/ images/stories/normativa/DM_6 luglio_2012_sf.pdf, 2012. [last access 24/12/2012]

Nielsen PH, Nielsen AM, Weidema BP, Dalgaard R, Halberg N. LCA food data base. www. lcafood.dk, 2003.

Patterson T, Esteves S, Dinsdale R, Guwy A. Life cycle assessment of biogas infrastructure options on a regional scale. Bioresour Technol 2011:15:7313-23.

Povellato A. Aumenta la superficie in affitto delle aziende professionali. Inf Agrar 2011;40: 32-9.

Scacchi C, González-García S, Caserini S, Rigamonti L. Greenhouse gases emissions and energy use of wheat grain-based bioethanol fuel blends. Sci Total Environ 2010;408(21):5010-8.

Smil V. Phosphorus in the environment: natural flows and human interferences. Annu Rev Energy Environ 2000;25:53-88. 
Tremblay N, Bouroubi Y, Bélec C, Mullen R, Kitchen N, Thomason W, et al. Corn response to nitrogen is influenced by soil texture and weather. Agron J $2012 ; 104(6): 1658-71$.

Uchida S, Hayashi K. Comparative life cycle assessment of improved and conventional cultivation practices for energy crops in Japan. Biomass Bioenergy 2012;36:302-15.
Vismara R, Malpei F, Centemero M. Biogas da rifiuti solidi urbani. Palermo: Flaccovio Editore; 2008.

Wulfa S, Jägerb P, Döhlerb H. Balancing of greenhouse gas emissions and economic efficiency for biogas-production through anaerobic co-fermentation of slurry with organic waste. Agr Ecosyst Environ 2006;112:178-85. 